\title{
CO-EVOLUTION OF LANGUAGE AND BEHAVIOUR IN AUTONOMOUS ROBOTS
}

\author{
SARA MITRI \\ Ecole Polytechnique Fédérale de Lausanne \\ EPFL-STI-I2S-LIS, Station 11 \\ CH-1015 Lausanne, Switzerland \\ sara.mitri@epfl.ch \\ PAUL VOGT \\ Language Evolution and Computation Research Unit, University of Edinburgh \\ 40 George Square, Edinburgh, EH8 9LL, UK \\ paulv@ling.ed.ac.uk
}

Computational studies on the evolution of language have often been criticised for the large amount of assumptions and simplifications they make. One particular criticism concerns the meaning of words (Ziemke \& Sharkey, 2000), which are often predefined (e.g., Kirby \& Hurford, 2002), or, in the case where they do develop ontogenetically, are typically unrelated to the agents' behavioural survival task (e.g., Vogt, 2003).

In an attempt to address these problems, this work explores the co-evolution and correlation between language use and behavioural learning in a realistic simulated environment of robotic agents, where a task must be solved to ensure survival. Experiments involving different environmental setups, population sizes and learning schemes are used to study the conditions under which language can emerge and stabilise and how the language affects the collective behaviour of the agents using it. The aim of the study is to investigate whether learning language together with simple survival skills can lead to an overhead in complexity, or can work as a tool for a more rapid emergence of increasingly intelligent behaviour, as well as a flexible, yet robust language.

The simulated Nomad 150 robots in this study are given a "survival task" of collecting red and blue balls and depositing them in a red or blue bin in return for energy. After a ball has been deposited, the agent must decide - using a reinforcement learner - which ball to collect next and where to take it, receiving a reward, depending on the amount of energy gained. If a ball is deposited in the bin of the opposite colour, no energy gain is received, otherwise the increase in energy is regulated by the environmental setup. Three environmental setups are 
used: a "cooperation" environment, in which two agents must deposit the same colour ball in the correct bin at the same time; a "division of labour" environment, where two agents must simultaneously deposit opposite colours in the correct bin; and a simple environment, where there is no need for collective action and energy is gained if an agent deposits a ball in the right bin. The robots must learn to coordinate their actions in order to achieve higher performance, which is an incentive for developing and using language. The evolved vocabulary was restricted to 8 wholistic utterances. The implications of using a horizontal model based on the language game model (Steels, 1997) as opposed to a vertical one based on the Iterated Learning Model (Kirby \& Hurford, 2002) are compared.

The results and their significance can be summarised in the following four points: (1) A perfect language with a fixed meaning space is not useful in every environment. (2) Where language is useful and a horizontal learning mechanism is used, a stable language evolves and leads to higher performance levels and faster behavioural learning. (3) A larger population size leads to an increase in language coherence, suggesting that language might evolve faster in large populations. (4) Even when a partially-stabilised language is evolved, the minimal performance is still sufficient for survival and is higher than that of non-communicating agents.

These results stress the difficulty of language development and stabilisation, but also show how in an environment where cooperation is highly beneficial, language can stabilise over time to help coordinate the behaviours of individual agents and improve the overall efficiency of a population. The interdependence of behavioural learning and language learning therefore helps to bootstrap both processes, leading to a higher performance in solving a survival task. The outcome of this study contributes to the field of language evolution by showing that language and behaviour can co-evolve as interdependent learning processes in a model where language has a function for survival, but also highlights the benefits of a bottom-up design for intelligent, autonomous and flexible robots that can survive in a dynamically changing environment through the use of a language that is developed during their lifetime according to a survival task.

\section{References}

Kirby, S., \& Hurford, J. (2002). The emergence of linguistic structure: An overview of the iterated learning model. In A. Cangelosi \& D. Parisi (Eds.), Simulating the evolution of language (p. 121-148).

Steels, L. (1997). The synthetic modeling of language origins. Evolution of Communication, 1(1), 1-34.

Vogt, P. (2003). Anchoring of Semiotic Symbols. Robotics and Autonomous Systems, 43(2-3), 109-120.

Ziemke, T., \& Sharkey, N. (2000). A Stroll through the Worlds of Robots and Animals: Applying Jakob von Uexküll's Theory of Meaning to Adaptive Robots and Artificial Life. 